\title{
El papel de los medios de comunicación en situaciones de crisis sanitaria. La percepción de la población en torno al control y las normas sociales durante la pandemia del COVID-19
}

\section{The role of media in health crisis scenarios. The perception of society on social control and norms during the COVID-19 pandemic}

Gorka Roman Etxebarriet*. Universidad del País Vasco. España.

gorka.roman@ehu.eus

$[\mathrm{CV}] \odot \mathrm{R}^{\circ}$

María Álvarez-Rementería Álvarez*. Universidad del País Vasco. España.

maria.alvarezrementería@ehu.eus

[CV] $10 \mathrm{R}^{\mathrm{G}}$

Elizabeth Pérez-Izaguirre*. Universidad del País Vasco. España.

elizabeth.perez@ehu.eus

[CV] 1 G

Maria Dosil Santamaria*. Universidad del País Vasco. España.

maria.dosil@ehu.eus

$[\mathrm{CV}]$ O $\mathrm{R}^{\mathrm{C}}$

Este trabajo no habría sido posible sin la participación de todas las personas que, a pesar de las adversidades derivadas de esta crisis sanitaria, han empleado su tiempo en contribuir a esta investigación.

Cómo citar este artículo / Referencia normalizada

Roman, G., Álvarez-Rementería, M., Pérez-Izaguirre, E. y Dosil, M. (2020). El rol de los Medios de Comunicación en Situaciones de Crisis Sanitaria. La Percepción de la Población en torno al Control y las Normas Sociales durante la Pandemia del COVID-19. Revista Latina de Comunicación Social, 78, 437-456. https://www.doi.org/10.4185/RLCS-2020-1484

\section{RESUMEN}

Introducción: El presente trabajo se centra en el análisis de la percepción de la población en torno al papel desempeñado por los medios de comunicación durante la crisis sanitaria derivada de la pandemia del COVID-19, y cómo las personas adoptan determinados roles orientados a la legitimación e implementación del control y las normas sociales. Metodología: Para la consecución del mismo, se diseñó y distribuyó una encuesta de tipo Likert que fue respondida por 704 personas de distintas regiones del Estado español. Los datos han sido posteriormente analizados mediante la

* Todas las autoras han colaborado por igual en la redacción de este artículo. 
herramienta de análisis estadístico SPSS. Resultados: Los resultados indican que el sexo y la edad fueron variables determinantes en la legitimación e implementación del control social entre iguales durante el confinamiento. Los resultados también han puesto en relieve la relación existente entre la forma en la que las personas perciben el rol de los medios de comunicación y su predisposición a acatar y ejercer el control y las normas sociales. Discusión y conclusiones: Tanto la literatura como los datos obtenidos para la realización del presente estudio, muestran la conexión existente entre el rol desempeñado por los medios de comunicación en situaciones de alarma social y de crisis sanitaria, y los procesos de construcción y legitimación de cosmovisiones de la "realidad" derivadas de la desinformación, los bulos y los ruidos informativos.

PALABRAS CLAVE: Medios de comunicación; control social; desinformación; crisis sanitaria; COVID-19.

\begin{abstract}
Introduction: The present study focuses on the analysis of the perception on the roles of mass media during the health crisis derived from the COVID-19 pandemic. It also studies how people adopt specific roles oriented towards the legitimation and implementation of control and social rules. Methodology: A questionnaire was designed based on a set of Likert type answers responded by 704 people from different regions in Spain. Data were analyzed using SPSS software package. Results: Results indicate that sex and age are determining variables in the legitimation and implementation of social control between peers. Results also indicate that there is a relationship between the way people perceive the role of media and their predisposition to abide and exercise social rules and control. Discussion and conclusions: Both the literature and obtained data show that there is a relationship between the role of media in situations of social alarm and health crisis, and the processes of legitimation of cosmovisions of "reality" derived from disinformation, hoaxes and noise information.
\end{abstract}

KEYWORDS: Media; social control; disinformation; health crisis; COVID-19.

\title{
CONTENIDO
}

1. Introducción. 1.1. Control social, normas sociales y vigilancia en situaciones de alarma social. 1.2. Medios de Comunicación y Control Social. 2. Objetivos. 3. Metodología. 3.1. Diseño y procedimiento. 3.2. Instrumento. 3.3. Participantes. 3.4. Análisis de datos. 4. Resultados. 4.1. Normas sociales en función del sexo y la edad. 4.2. Control social en función de la edad. 4.3. Medios de comunicación en función de la edad. 4.4. Nivel de ansiedad y miedo en función de la edad. 4.5. Asociaciones entre variables normas sociales, control social y medios de comunicación. 5. Discusión. 6. Conclusiones. 7. Bibliografía. 8. Currículum Vitae.

\section{Introducción}

En diciembre de 2019 se produce un brote de una desconocida enfermedad respiratoria en Wuhan (provincia de Hubei, China) que se extendió por todo el país, acaparando la atención del mundo entero (Wang et al., 2020). La preocupación internacional se centra en una nueva cepa de coronavirus, SARS-CoV-2, con una elevada capacidad de transmisión entre personas. La enfermedad, denominada COVID-19, provoca una sintomatología especialmente peligrosa para personas de avanzada edad o con patologías previas.

Un mes después de su descubrimiento, el 30 de enero de 2019, la Organización Mundial de la Salud (OMS) declaró una emergencia de salud pública mundial (Guo et al., 2020), instando a todos los 
países afectados a tomar medidas para contener y frenar la propagación del virus (Grasselli, Pesenti y Cecconi, 2020). Así, las autoridades sanitarias locales comenzaron a establecer diferentes medidas de contención basadas en estrategias muy variadas y adaptadas a cada contexto. En China, el principal foco del brote, las autoridades sanitarias iniciaron una campaña que incluyó, en un principio, el aislamiento de personas infectadas, el monitoreo de la ciudadanía y la recopilación de datos de los pacientes para caracterizar y controlar la enfermedad (Wang et al., 2020). Mientras tanto, en Europa, la propagación del virus se produjo de forma muy desigual en los distintos países, teniendo los peores escenarios en Italia, donde se implementaron las primeras medidas de confinamiento domiciliario de toda la población en Europa y, poco después, en el Estado español (Yuan et al., 2020).

El 15 de marzo de 2019, el Gobierno español declaró la emergencia nacional, estableciendo el confinamiento total de la población durante 15 días (Legido-Quigley et al., 2020), que posteriormente se prolongó hasta el 21 de junio de 2020. En este escenario, la centralización de la gestión de la crisis sanitaria en un Estado formado por 17 Comunidades Autónomas muy diversas, ha generado una gran controversia. En respuesta a la propagación del virus, el Gobierno español aprobó el Real Decreto (463/2020) para asumir nuevos poderes y competencias en materia de sanidad, transporte y asuntos internos, incluyendo las referentes a las fuerzas armadas y cuerpos de seguridad del Estado (Legido-Quigley et al., 2020). A lo largo y ancho de todo el territorio nacional fueron implantadas distintas medidas de distanciamiento social y restricciones en los desplazamientos de las personas.

Además de una preocupación generalizada por la enfermedad y sus consecuencias sanitarias, varios estudios se han hecho eco de las repercusiones sociales de la pandemia, especialmente en términos de salud mental durante y más allá del confinamiento (Berasategi et al., 2020; Ozamiz, Dosil, Idoiaga y Picaza, 2020), las consecuencias psicológicas a largo plazo, como el miedo (Pakpuor y Griffiths, 2020) o el cambio de comportamiento social a gran escala que esta situación ha provocado en todo el mundo (Van Bavel et al., 2020).

El presente artículo pretende poner en valor el papel de los medios de comunicación como herramienta de control social (Roman, 2018) en situaciones de crisis sanitaria global. Los medios de comunicación son instrumentos que legitiman la normativización social, cultural y política por su capacidad de definir y establecer socialmente lo que es correcto y lo que no (Juris, 2003). De esta forma, a menudo reproducen aquellos significados que obedecen a los intereses dominantes (Bowman, 2003; Herman y Chomsky, 1988). Partiendo de los datos obtenidos de las encuestas llevadas a cabo en varias regiones del Estado español, se realiza una reflexión en torno a las estrategias de control social, a las normas sociales y al rol de los medios de comunicación durante la crisis del COVID-19.

\subsection{Control social, normas sociales y vigilancia en situaciones de alarma social}

Tal y como muestra la bibliografía, durante situaciones de excepción asociadas a alarmas de tipo sanitario, conflictos armados o catástrofes naturales entre otras, los gobiernos e instituciones gubernamentales han hecho tradicionalmente uso de estrategias y herramientas orientadas a fomentar el control social (Roman, 2015; Bowman, 2001; Juris, 2008). El control social es un concepto ampliamente estudiado desde distintas disciplinas (Cohen, 1985), y por tanto difícil de acotar a un solo campo de investigación. Desde la sociología, Giddens y Sutton (2015) lo definen como "todos los mecanismos formales e informales, así como los controles internos y externos que operan para producir conformidad" (p. 241). Según Ross (2017), los sistemas de control social pueden estar organizados en relación a dos aspectos: la moralidad, que tiene que ver con la influencia de las 
expresiones sociales y culturales; y lo organizativo o de corte más normativo, lo que el autor define como lo político. Castillo (2015) añade, además, que son las estrategias que utiliza un individuo o grupo con cierta autoridad, las que definen que es el control social. La autoridad, en el supuesto que nos ocupa, se referiría a los cuerpos policiales y militares de un Estado o una región que estén oficialmente autorizados a ejercer dicho control. Las estrategias que determinan el control social que imponen estos cuerpos, se establecerían por lo tanto para mantener un determinado tipo de orden social.

Según Swader (2017), el control social es el modo en que las normas sociales influyen en el comportamiento de las personas, de manera que el comportamiento de estas viene marcado por procedimientos burocráticos, normas y el poder policial. Otras estrategias de control social incluyen también el uso de sistemas de control como las cámaras de vídeo vigilancia o los Sistemas de Posicionamiento Global (Global Positioning System, GPS). El GPS puede ser utilizado para medir y controlar los movimientos y desplazamientos de una población en concreto (Sheikh, Sheikh y Sheikh, 2020). En ciertas situaciones, dichos datos pueden ser utilizados por los gobiernos para monitorear si una población o determinada persona ha cumplido con su cometido cívico, a través de las fuerzas de seguridad del estado. Estudios como el de Bevan (2019), que se centra en los sistemas de vigilancia que adoptan las instituciones escolares en la "era de los tiroteos masivos", reflejan esta tendencia hacia la vigilancia de flujos, que le gana terreno a las tradicionales medidas como el control de metales o el aumento de la presencia policial. Con el paso del tiempo, la normalización de dichas estrategias de control por parte de la población promueve, según Laca (2011), una "cultura basada en el miedo", que es utilizada como herramienta de poder y control social.

Ashcroft, Griffiths y Tiffin (2006) añaden que dicho control social es posible gracias al lenguaje, ya que este permite describir la realidad a través de determinadas cosmovisiones. Es decir, a través del lenguaje en sus diferentes formas, tales como el lenguaje verbal o el no verbal, refiriéndonos en este caso a aquel que puede incluir gestos ritualizados y performativos (Bloch, 1975, 1989, 1998) e incluso el uso de la fuerza física, se puede transmitir un mensaje de dominación por parte de las fuerzas autorizadas para ejercer cierto tipo de poder y violencia de carácter performativo (Juris, 2003). De este modo, siguiendo los marcos legales, los cuerpos y fuerzas de un Estado o región pueden ejercer dicho poder bajo el pretexto de velar por el cumplimiento de las normas de una determinada sociedad (Juris, 2008). Este velar implica, en este caso, el uso de un determinado tipo de lenguaje que ejerce un poder desde la legislación hacia la ciudadanía de a pie. Cuerpos como la policía o el ejército estarían legitimados para ejercer dicho poder y tendrían la potestad para sancionar a los ciudadanos que no cumplan con la legislación que el Estado o región haya marcado.

Una de las cuestiones más polémicas con respecto al control social está ligada a la justificación de las normas y del propio sistema autorizado para ejercer ese control social (Gau, 2015). Chakraborty (2020) también hace referencia a este hecho centrándose en el proceso de amplificación social de los medios de comunicación durante la pandemia del COVID-19. Como se verá en las siguientes secciones, los medios de comunicación habrían contribuido a que el riesgo percibido por la población sea en ocasiones poco ajustado a la realidad. Además, las políticas que se han puesto en marcha alrededor del mundo, no han estado adecuadamente informadas en muchos casos. La intersección entre la desinformación y la falta de rigor científico en muchas de las decisiones políticas y las medidas de control social propuestas por ciertos países, habrían dado lugar a una percepción social de la pandemia que no se correspondería con los hechos que se estaban viviendo. Igartua, OrtegaMohedano y Arcila-Calderón (2020), señalan por otro lado, que la comunicación en general ha resultado esencial para la transmisión de información preventiva en torno al Coronavirus. No obstante, las redes sociales de tipo visual como Instagram, Facebook y Youtube no habrían tenido un impacto destacable en ese sentido. Por el contrario, Ramón Fernández (2020) pone de relieve la 
influencia negativa de la desinformación y las fake news durante la pandemia, así como las consecuencias sociales de las mismas. En última instancia, la autora aboga por la aprobación de una legislación en contra las fake news para evitar este tipo de problemas.

Otros estudios relativos a la pandemia del COVID-19 se centran en aspectos relacionados con el control social que tienen que ver con el cumplimiento de las normas sanitarias que imponen los gobiernos. Este es el caso de Sibley et al. (2020) en un estudio llevado a cabo en Nueva Zelanda. Los autores analizaron las percepciones de una muestra de 1003 individuos antes y después del confinamiento durante la pandemia del COVID-19. Los resultados indican que cuando la población confía en las medidas de control social adoptadas por el Estado y considera el virus como un enemigo común, se genera un sentimiento de comunidad que ayuda al establecimiento de relaciones afectivas y a la resiliencia. Además, tal y como señalan Blair, Morse y Tsai (2017) en su estudio sobre el compromiso ciudadano con las medidas establecidas para combatir el Ébola en Liberia, la confianza en los gobiernos locales también ayudaría a la adherencia a las medidas sanitarias de control social. Asimismo, de acuerdo con Gino, Norton y Weber (2016), el compromiso de la población con las medidas adoptadas en situaciones de emergencia se ve incrementado cuando la población se considera capaz de superar la crisis y, además, ven que su comportamiento tiene un impacto directo positivo en otras personas, así como en la mejora de la situación.

El control social al que nos hemos referido en el presente apartado tendría además un especial impacto en una serie de colectivos que se encuentran en una situación de mayor vulnerabilidad, como es el caso de las mujeres, los migrantes y las personas sin recursos, entre otros. En este sentido, estudios publicados recientemente subrayan que las mujeres están expuestas, en mayor medida que los hombres, a sufrir las consecuencias derivadas de este tipo de situaciones extremas (Bradshaw, 2015; McLaren, Wong, Nguyen y Mahamadachchi, 2020). Estas consecuencias están ligadas a la estructura social, especialmente en el marco de los cuidados, que sitúa a los sujetos que los ejercen, en su mayoría mujeres, ante un mayor riesgo de contagio por la enfermedad COVID-19 (Wenham, Smith y Morgan, 2020) y ante una mayor exposición a la vigilancia y otras estrategias de control, ya que las tareas vinculadas a los cuidados precisan de un mayor número de desplazamientos fuera del hogar para el abastecimiento de los bienes esenciales o el acompañamiento a servicios médicos y otras utilidades. Varios estudios de carácter estadístico llevados a cabo por el INE (Instituto Nacional de Estadística) dan fe de ello. Por ejemplo, en los datos referidos al cuidado de personas dependientes, son el 8,6\% de las mujeres frente al 5,1\% de los hombres las que ejercen estas labores (INE, 2020). En el caso de las familias monoparentales, aquellas a cargo de mujeres, cuatriplicarían a las que están a cargo de hombres (INE, 2019). Además, en palabras de Wenham, Smith y Morgan (2020), en situaciones excepcionales como la actual, esta carga se habría visto aumentada de un modo desproporcionado.

Gausman y Langer (2020) señalan que las medidas tomadas por los gobiernos han pasado por encima cualquier consideración en este sentido, no estableciendo políticas de conciliación para evitar la carga adicional sobre las mujeres. Esto habría llevado a un mayor desgaste emocional y un empeoramiento del bienestar general de las mujeres durante la pandemia (McLaren, Wong, Nguyen y Mahamadachchi, 2020).

\subsection{Medios de Comunicación y Control Social}

El control social y el rol desempeñado por los medios de comunicación ha sido un elemento de análisis recurrente en el seno de las diversas disciplinas académicas durante gran parte del siglo XX y del siglo XXI (Ashcroft, Griffiths y Tiffin, 2006). Los expertos han acudido habitualmente al estudio de casos que han resultado paradigmáticos y altamente representativos en referencia al rol de 
los medios de comunicación y el control social, basándose en experiencias pretéritas y actuales todas ellas altamente significativas en cuanto a la influencia de los medios de comunicación y sus consecuencias más directas e inmediatas (Roman, 2018).

La utilización interesada de los medios de comunicación y el control sobre estos para su implementación como herramientas de control social, se ha dado tradicionalmente durante situaciones de conflictos armados, especialmente en aquellos lugares donde el componente etnocultural ha sido un elemento clave (Roman, 2015, 2018), o durante situaciones de alarma social o crisis sanitaria donde la información ha sido empleada como una herramienta también de control social. En este contexto, la desinformación y la manipulación de la información se erigen como efectivas herramientas de control social que a menudo son utilizadas de un modo organizado en aras de lograr una serie de objetivos de tipo económico, social, cultural o político. En palabras de Rodríguez (2018), la desinformación es un modo de manipulación organizado, estructurado y planificado minuciosamente, que responde a una estrategia concreta y a unos objetivos políticos muy claros”. Rodríguez-Fernandez $\left(2019^{\mathrm{a}}, 2019 \mathrm{~b}\right)$ también indica en este sentido que la desinformación y las fake news representan en el presente uno de los grandes problemas en torno a los medios de comunicación y el control social. Rodríguez-Fernández (2019b) también señala que esos problemas son especialmente visibles en aquellos contextos donde se intenta desprestigiar al adversario político mediante campañas de propaganda construidas a partir de bulos, ruidos informativos y todo tipo de desinformación en general. Este tipo de desinformación se agravaría, según Salaverría, Buslón, López-Pan, León, López-Goñi y Erviti (2020), en situaciones de pandemia como la actual derivada del COVID-19, a través de la implementación de bulos de carácter informativo, catalogados por estos mismos autores en cuatro categorías según la gravedad: broma, exageración, descontextualización y engaño. La influencia y consecuencias en el seno de la sociedad de cada una de estas categorías, se fundamentaría en "el volumen y distribución real de cada uno de esos tipos de bulos, sin el tamiz de las plataformas de verificación” (p. 13).

En palabras de Gitlin (1980), las fuerzas de seguridad y los gobiernos pueden manipular ciertas imágenes y situaciones, y reinsertarlas en nuevos contextos donde determinados movimientos y actitudes se muestran como peligrosas y subversivas. Los medios de comunicación se habrían convertido, pues, en sistemas cardinales para la distribución de ideología.

Según Juris (2003) los medios de comunicación son efectivos instrumentos para la normativización social, cultural y política ya que son capaces de dictar de un modo altamente efectivo qué comportamientos han de ser aceptados y cuáles denostados. En este sentido, los medios de comunicación estarían activamente comprometidos en la producción de realidad social basándose en sus propios intereses y objetivos informativos. En opinión de Juris (2008), las élites económicas estarían detrás de los discursos mediáticos mediante la concentración de la propiedad de los medios, el sistema de entretenimiento, los vínculos con expertos del gobierno y los negocios. Debido a estos poderosos filtros ideológicos, los medios de comunicación tenderían a reproducir los significados culturales que se ajustan a los intereses dominantes de la sociedad mediante la construcción de determinadas retóricas de la identidad (Bowman, 2001, 2003) que establecerán cuáles son los límites que la ciudadanía no podrá traspasar.

Los medios de comunicación del presente pueden considerarse como la herramienta más eficiente y masiva de intercambio unidireccional de información (Roman, 2015), dando pie a una hegemonía en torno a la difusión de la información que se habría agudizado aún más durante las últimas décadas. Autores como Golovina (2014) también se hacen eco del protagonismo de los medios de comunicación en el desarrollo de aspectos tales como la difusión de la información de un modo inmediato y masivo. Castillo (2009), por otro lado, afirma que los medios de comunicación se han 
convertido en gran medida en fuentes de poder que responderían a los intereses de la hegemonía política, social o económica del momento. Ostentar el control sobre los grandes medios de comunicación derivaría en multitud de ocasiones en la instauración y reproducción sistemática de cosmovisiones y sistemas de valores que responderían a intereses ocultos de los propietarios de estos, y que en multitud de ocasiones podrían traducirse además en la constitución simbólica de adversarios ideológicos reales o ficticios (Bowman, 2001, 2003) y de otredades políticas, sociales o etnoculturales (Hage, 1992).

En palabras de Stuart Hall (1982) los medios de comunicación se encargan de seleccionar, presentar, estructurar y moldear la información que transmiten. De este modo atribuirían significado a las cosas (Gitlin, 1980). Las herramientas mediáticas se fundamentarían, pues, en una serie de patrones de conocimiento e interpretación, que serían los garantes de ciertos símbolos orientados a organizar discursos, de carácter tanto verbal como visual.

Los marcos mediáticos hegemónicos tienden a cuestionar la protesta social y las divergencias que pongan en cuestión el Status Quo social estandarizado, particularmente cuando éstas cuestionan sus presupuestos básicos sobre el mercado y el Estado, como algo protagonizado por individuos peligrosos y criminales (Juris, 2003). No debemos obviar, en este sentido, que las retóricas de carácter dualista son efectivos mecanismos para el control social (Yong Tao, 2000) donde esos mismos imaginarios dualistas que enfrentan al "mal" y al "mundo civilizado" o al nosotros y al ellos, no dejan lugar a un pensamiento o posición neutral. Cualquier tipo de disidencia que cuestione los discursos hegemónicos y estandarizados de los medios de comunicación, sería susceptible de ser considerada como un ataque al Status Quo y al Nosotros etno-cultural hegemónico, con el consecuente peligro de ser socialmente estigmatizada. Una vez constituidos los enemigos de la hegemonía social, ésta se refuerza con la excusa de luchar contra aquello que es peligroso y que amenaza el bienestar de la sociedad o comunidad en cuestión.

Roman (2015) recuerda que los medios de comunicación estarían en gran medida detrás de esa estigmatización a la que nos referimos y que afectaría directamente a ciertas personas y grupos sociales. Estos estarían en multitud de ocasiones detrás de la construcción y difusión de estereotipos, prejuicios e ideas estigmatizadoras en torno a ciertos colectivos en situación de vulnerabilidad o que hayan optado por oponerse al mainstream sociopolítico y etno-cultural. Y es que los medios de comunicación contarían con un poder performativo (Austin, 1982; Bourdieu, 1985) capaz de desfigurar la "realidad" de ciertos contextos y colectivos, difundiendo informaciones que alimentarían el miedo y el rechazo hacia ciertas ideas y personas. Esta misma idea ha sido recogida en distintos estudios que destacan el componente etnocéntrico asociado a las enfermedades infecciosas (Van Bavel et al., 2020), como fue el caso de la plaga bubónica (Cohn, 2012) durante la cual el origen o clase social fueron motivo de múltiples asesinatos en toda Europa; o como ya se ha recogido en el caso del Coronavirus (Russell, 2020), habiendo constancia de ataques físicos en contra de la comunidad asiática, o declaraciones realizadas desde diversas instancias gubernamentales que se han referido a la enfermedad COVID-19 como "la enfermedad de Wuhan" o el "virus chino".

En tiempos de crisis sanitaria, el componente identitario estaría directamente relacionado con la respuesta cooperativa. El apoyo entre iguales y la preocupación por el "otro" es considerablemente mayor cuando existen lazos identitarios (Drury, Cocking y Reicher, 2009). En este sentido, el papel de los medios de comunicación es crucial, ya que pueden llegar a perturbar dicha predisposición y generar una respuesta individualista, como ha ocurrido con la exhibición de imágenes de estanterías vacías en los supermercados, lo que provocó una percepción errónea y amenazante sobre una supuesta falta de abastecimiento (Van Bavel et al., 2020). 


\section{Objetivos}

El objetivo general de este estudio es analizar la percepción de la población en torno al rol desempeñado por los medios de comunicación durante la crisis sanitaria derivada de la COVID-19 en relación a la predisposición que tiene la población para acatar y reproducir estrategias de control social.

Los objetivos específicos que se desprenden de dicho propósito general son los siguientes:

- Indagar en el papel de los medios de comunicación y su influencia sobre la población del Estado español en la legitimación e implementación del control social entre iguales durante estado de alarma sanitaria.

- Explorar la percepción de las personas en torno al control y las normas sociales asociadas a la influencia de los medios de comunicación en función de las variables sexo y edad.

- Describir los procesos mediante los que los medios de comunicación construyen "realidad" social y cosmovisiones específicas a partir de la desinformación y del ruido informativo.

\section{Metodología}

Para poder alcanzar los objetivos previamente expuestos, se diseñó un cuestionario dirigido a la sociedad en general que permitiese investigar la relación entre los medios de comunicación, el control social y la percepción de ciertas normas sociales durante la pandemia del COVID-19.

\subsection{Diseño y procedimiento}

Los cuestionarios fueron enviados mediante la plataforma Twitter del grupo de investigación de KideON (@kide_on) a diferentes asociaciones del tercer sector y a la población en general. En este tweet se les invitaba a participar en el estudio a través de un enlace en el que se podía cumplimentar el cuestionario. Una vez realizada la encuesta, la persona invitada no tenía posibilidad de entrar de nuevo al cuestionario certificando, de esta manera, que únicamente se pudiese hacer un cuestionario por persona. El muestreo fue probabilístico para que todas las personas tuviesen la oportunidad de participar en el estudio y garantizar así la aleatorización.

Los cuestionarios fueron contestados por un total de 952 individuos. Después de analizar la base de datos en Microsoft Excel (https://products.office.com/), los cuestionarios mostraron un patrón de falta de respuestas de más del $50 \%$ en varios bloques. En consecuencia, se optó por excluir todos los cuestionarios con menos del 50\% de los ítems completados, y así se retiraron 248 cuestionarios de la muestra. El estudio cumplió con todas las disposiciones de la Ley 15/1999 de Protección de Datos Personales, además, informaban del carácter voluntario de la participación y de la necesidad que hay de que los y las participantes den su consentimiento antes de comenzar a responder el cuestionario.

El cuestionario fue publicado a fecha de 1 de abril de 2020, coincidiendo con la primera prórroga del estado de alarma tras las dos semanas que se decretaron al comienzo de la crisis sanitaria en el Estado español, y tercera semana de confinamiento domiciliario. La última respuesta registrada fue del 23 de mayo de 2020, poco antes de alcanzar el ecuador del periodo total de confinamiento.

\subsection{Instrumento}

Cuestionario ad hoc. Este instrumento reúne información sobre la edad de los y las participantes, el sexo, nacionalidad, enfermedad crónica y posible contacto o no con personas infectadas por el COVID-19. 
Respecto al instrumento de la investigación, se diseñó un cuestionario compuesto por 43 ítems, divididos en 3 bloques temáticos. El primer bloque estaba compuesto por 17 ítems, que describen las normas sociales con respuestas dicotómicas. El segundo bloque estaba compuesto por 11 ítems relacionados con los derechos y el control social. El último bloque estaba dirigido a los medios de comunicación y la alarma social, para lo que se diseñaron 15 ítems. Ambos bloques son analizados con respuestas tipo Likert desde el 0 (muy en desacuerdo) hasta el 5 (muy de acuerdo). Para este estudio se analizó únicamente la respuesta tipo Likert 3 (un poco de acuerdo). Es por ello por lo que los resultados no indican el $100 \%$ de los casos, ya que los casos restantes están indicando otras respuestas de tipo Likert.

\subsection{Participantes}

El total de participantes en este estudio fueron 704 personas de distintas regiones del Estado español de las cuales el $75.3 \%(n=530)$ eran mujeres y el $24.7 \%(n=174)$ eran hombres. En relación con la edad, el 16.2\% ( $n=114)$ eran jóvenes de entre 18 y 25 años, un $42.9 \%(n=302)$ eran personas de entre 26 y 39 años, un $30.8 \%(n=217)$ personas adultas de entre 40 y 55 años y por último, un $10.1 \%$ $(\mathrm{n}=71)$ mayores de 56 años $(M=37.9 ; D T=14.9)$, siendo el participante de menor edad de 18 años y el de mayor edad de 74 años.

\subsection{Análisis de datos}

Para el análisis de los datos se utilizó el programa estadístico SPSS v.25 (INC., Chicago, IL). Se realizaron análisis descriptivos de las variables sociodemográficas, chi cuadrados y análisis del tamaño del efecto entre las variables.

\section{Resultados}

\subsection{Normas sociales en función del sexo y la edad}

En la Tabla 1 se muestran las preguntas que fueron respondidas afirmativamente por los y las participantes de este estudio. Concretamente, las personas que respondieron sí creer que el confinamiento en su domicilio era necesario para superar la pandemia del coronavirus, mostraron una asociación estadísticamente significativa, $x 2=4.70, p=.10$, Vcramer $=.08$, con un tamaño del efecto pequeño con el sexo, siendo un $72 \%(n=507)$ mujeres y un $22.6 \%(n=159)$ hombres. Asimismo, las personas participantes indicaron que sí creían que controlando a la gente que incumple el confinamiento se ayuda a la sociedad, mostrando una asociación estadísticamente significativa con el sexo, $x 2=12.18, p=.001$, Vcramer $=.13$, siendo $13.9 \%(\mathrm{n}=98)$ las mujeres que respondieron sí, y $7.7 \%(\mathrm{n}=54)$ los hombres.

Tabla 1. Normas Sociales en función del sexo

\begin{tabular}{|c|c|c|c|c|}
\hline \multirow[t]{2}{*}{ Normas sociales } & \multicolumn{2}{|c|}{$n(\%)$} & \multirow[t]{2}{*}{$X 2$} & \multirow[t]{2}{*}{ Vcramer } \\
\hline & mujer & hombre & & \\
\hline $\begin{array}{l}\text { Creo que el confinamiento en mi domicilio es necesario para } \\
\text { superar la pandemia del Coronavirus. }\end{array}$ & $\begin{array}{c}72 \% \\
(\mathrm{n}=507)\end{array}$ & $\begin{array}{c}22.6 \% \\
(\mathrm{n}=159)\end{array}$ & $4.70 *$ & .08 \\
\hline $\begin{array}{l}\text { Creo que controlando a la gente que incumple el confinamiento } \\
\text { estoy ayudando a la sociedad. }\end{array}$ & $\begin{array}{l}13.9 \% \\
(\mathrm{n}=98)\end{array}$ & $\begin{array}{l}7.7 \% \\
(\mathrm{n}=54)\end{array}$ & $12.18 * * *$ & .13 \\
\hline $\begin{array}{l}\text { Creo que la sociedad en general quiere que recrimine o denuncie a } \\
\text { personas que incumplen el confinamiento. }\end{array}$ & $\begin{array}{l}26.6 \% \\
(\mathrm{n}=187)\end{array}$ & $\begin{array}{l}10.9 \% \\
(\mathrm{n}=77)\end{array}$ & $4.50 *$ & .08 \\
\hline
\end{tabular}

Fuente: Elaboración propia. 
En función a la edad y las normas sociales, las personas que respondieron sí a las preguntas: creo que controlando a la gente que incumple el confinamiento estoy ayudando a la sociedad; creo que los políticos quieren que recrimine o denuncie a personas que incumplen el confinamiento; creo que la policía quiere que recrimine o denuncie a personas que incumplen el confinamiento y; creo que la sociedad en general quiere que recrimine o denuncie a personas que incumplen el confinamiento; mostraron asociaciones estadísticamente significativas, siendo las personas con edades de entre 26 y 39 años los que indicaron un porcentaje superior (Ver Tabla 2).

Tabla 2. Normas Sociales en función de la edad

\begin{tabular}{|c|c|c|c|c|c|c|}
\hline \multicolumn{7}{|l|}{ Normas sociales } \\
\hline & $18-25$ & 26-39 & $40-55$ & $>56$ & $X 2$ & Vcramer \\
\hline $\begin{array}{l}\text { Creo que controlando a la gente que incumple } \\
\text { el confinamiento estoy ayudando a la sociedad }\end{array}$ & $\begin{array}{c}2.1 \% \\
(\mathrm{n}=15)\end{array}$ & $\begin{array}{c}8 \% \\
(\mathrm{n}=56)\end{array}$ & $\begin{array}{l}7.7 \% \\
(\mathrm{n}=54)\end{array}$ & $\begin{array}{c}3.8 \% \\
(\mathrm{n}=27)\end{array}$ & $19.17 * * *$ & .17 \\
\hline $\begin{array}{l}\text { Creo que los políticos quieren que recrimine o } \\
\text { denuncie a personas que incumplen el } \\
\text { confinamiento }\end{array}$ & $\begin{array}{c}7.7 \% \\
(n=54)\end{array}$ & $\begin{array}{l}13.5 \% \\
(n=95)\end{array}$ & $\begin{array}{l}10.2 \% \\
(n=72)\end{array}$ & $\begin{array}{c}3.7 \% \\
(n=26)\end{array}$ & $9.71 *$ & .12 \\
\hline $\begin{array}{l}\text { Creo que la policía quiere que recrimine o } \\
\text { denuncie a personas que incumplen el } \\
\text { confinamiento }\end{array}$ & $\begin{array}{c}9.2 \% \\
(n=65)\end{array}$ & $\begin{array}{c}16.2 \% \\
(n=114)\end{array}$ & $\begin{array}{l}11.9 \% \\
(n=84)\end{array}$ & $\begin{array}{c}4.1 \% \\
(n=29)\end{array}$ & $13.77 * *$ & .08 \\
\hline $\begin{array}{l}\text { Creo que la sociedad en general quiere que } \\
\text { recrimine o denuncie a personas que } \\
\text { incumplen el confinamiento }\end{array}$ & $\begin{array}{c}8.4 \% \\
(n=59)\end{array}$ & $\begin{array}{l}14.1 \% \\
(n=99)\end{array}$ & $\begin{array}{l}11.1 \% \\
(n=78)\end{array}$ & $\begin{array}{c}4 \% \\
(n=28)\end{array}$ & $13.09 * *$ & .14 \\
\hline
\end{tabular}

Fuente: Elaboración propia.

\subsection{Control social en función de la edad}

Las personas que respondieron estar un poco de acuerdo con implementar medidas de control más estrictas, aunque eso suponga que el Gobierno y cuerpos policiales tengan más acceso a su vida privada, mostraron una asociación estadísticamente significativa con la edad, $x 2=19.17, p=.001$, Vcramer $=.17$, con un tamaño del efecto pequeño. Asimismo, se mostró una asociación estadísticamente significativa con la edad en las personas que respondieron estar un poco de acuerdo con que otros grupos políticos habrían gestionado mejor la crisis, $x 2=9.71, p=.005$, Vcramer $=.12$, con un tamaño del efecto de nuevo pequeño. De nuevo en este caso, el rango de edad con porcentajes superiores se da entre los 26 años y los 39 años.

Tabla 3. Control Social en función de la edad

\begin{tabular}{lcccccc}
\hline Control social & $\mathbf{1 8 - 2 5}$ & $\mathbf{2 6 - 3 9}$ & $\mathbf{4 0 - 5 5}$ & $\mathbf{> 5 6}$ & X2 & Vcramer \\
\hline $\begin{array}{l}\text { Creo que se deben implementar medidas de control } \\
\text { más estrictas, aunque eso suponga que el Gobierno y } \\
\text { cuerpos policiales tengan acceso a mi vida personal. }\end{array}$ & $\begin{array}{c}2.1 \% \\
(\mathrm{n}=15)\end{array}$ & $\begin{array}{c}8 \% \\
(\mathrm{n}=56)\end{array}$ & $\begin{array}{c}7.7 \% \\
(\mathrm{n}=54)\end{array}$ & $\begin{array}{c}3.8 \% \\
(\mathrm{n}=27)\end{array}$ & $19.17 * *$ & .17 \\
$\begin{array}{l}\text { Creo que otros grupos políticos habrían gestionado } \\
\text { mejor la crisis. }\end{array}$ & $\begin{array}{c}7.7 \% \\
(\mathrm{n}=54)\end{array}$ & $\begin{array}{c}13.5 \% \\
(\mathrm{n}=95)\end{array}$ & $\begin{array}{c}10.2 \% \\
(\mathrm{n}=72)\end{array}$ & $\begin{array}{c}3.7 \% \\
(\mathrm{n}=26)\end{array}$ & $9.71 *$ & .12 \\
\hline
\end{tabular}

Nota $* * * p<.001 * * p<.01 ; * p<.05$

Fuente: Elaboración propia.

\subsection{Medios de comunicación en función de la edad}

En cuanto a los ítems sobre los medios de comunicación en tiempos de pandemia, se han encontrado asociaciones estadísticamente significativas con los diferentes rangos de edad (desde 18 años hasta mayores de 56 años), dándose el estadístico mayor en el siguiente ítem: creo que los medios de comunicación informan tanto sobre el Coronavirus para que la sociedad sienta miedo, $x 2=39.20$, $p=.001$, Vcrame $r=.14$ (Ver Tabla 4). 
Tabla 4. Medios de Comunicación en función de la edad

\begin{tabular}{|c|c|c|c|c|c|c|}
\hline \multicolumn{7}{|l|}{ Medios de comunicación } \\
\hline & $18-25$ & $26-39$ & $40-55$ & $>56$ & $X 2$ & Vcramer \\
\hline $\begin{array}{l}\text { Creo que los medios de comunicación informan tanto } \\
\text { sobre el Coronavirus para que la sociedad sienta miedo. }\end{array}$ & $\begin{array}{l}3.7 \% \\
(\mathrm{n}=26)\end{array}$ & $\begin{array}{l}11.5 \% \\
(\mathrm{n}=81)\end{array}$ & $\begin{array}{c}8 \% \\
(\mathrm{n}=56)\end{array}$ & $\begin{array}{l}3.3 \% \\
(\mathrm{n}=23)\end{array}$ & $39.20 * * *$ & .14 \\
\hline $\begin{array}{l}\text { Creo que los medios de comunicación deberían informar } \\
\text { más sobre otras noticias que no tengan que ver con el } \\
\text { Coronavirus. }\end{array}$ & $\begin{array}{c}4.1 \% \\
(n=29)\end{array}$ & $\begin{array}{l}11.6 \% \\
(n=82)\end{array}$ & $\begin{array}{c}8.2 \% \\
(n=58)\end{array}$ & $\begin{array}{c}4 \% \\
(n=28)\end{array}$ & $38.85 * * *$ & .14 \\
\hline $\begin{array}{l}\text { Creo que los medios de comunicación están siendo } \\
\text { sensacionalistas con el tratamiento que están haciendo de } \\
\text { la pandemia del Coronavirus. }\end{array}$ & $\begin{array}{c}5.3 \% \\
(n=37)\end{array}$ & $\begin{array}{c}9.2 \% \\
(n=65)\end{array}$ & $\begin{array}{l}10.1 \% \\
(\mathrm{n}=71)\end{array}$ & $\begin{array}{c}2.8 \% \\
(n=20)\end{array}$ & $38.45 * * *$ & .14 \\
\hline $\begin{array}{l}\text { Creo que los medios de comunicación informan tanto } \\
\text { sobre el Coronavirus para que la sociedad esté más } \\
\text { preocupada de lo normal. }\end{array}$ & $\begin{array}{c}4.4 \% \\
(n=31)\end{array}$ & $\begin{array}{l}13.1 \% \\
(n=92)\end{array}$ & $\begin{array}{c}8.4 \% \\
(n=59)\end{array}$ & $\begin{array}{c}2.8 \% \\
(n=20)\end{array}$ & $30.75 * *$ & .12 \\
\hline $\begin{array}{l}\text { Creo que los medios de comunicación compiten entre sí } \\
\text { para ver quien da la información más sensacionalista y } \\
\text { obtener así un mayor número de telespectadores. }\end{array}$ & $\begin{array}{c}3.8 \% \\
(n=27)\end{array}$ & $\begin{array}{c}8.5 \% \\
(n=60)\end{array}$ & $\begin{array}{c}7.5 \% \\
(n=53)\end{array}$ & $\begin{array}{c}2.6 \% \\
(n=18)\end{array}$ & $26.03 *$ & .11 \\
\hline
\end{tabular}
Nota***p<.001**p<.01; *p<.05

Fuente: Elaboración propia.

\subsection{Nivel de ansiedad y miedo en función de la edad}

La Tabla 5 muestra las asociaciones estadísticamente significativas en la respuesta un poco de acuerdo con las variables relacionadas con la ansiedad y el miedo en función del rango de edad. En las categorías de edad de entre 26 y 55 años se pueden apreciar los porcentajes más altos en comparación con los otros grupos de edad.

Tabla 5. Ansiedad y miedo en función de la edad

\begin{tabular}{|c|c|c|c|c|c|c|}
\hline \multicolumn{7}{|l|}{ Ansiedad y miedo } \\
\hline & $18-25$ & $26-39$ & $40-55$ & $>56$ & $X 2$ & Vcramer \\
\hline Estar confinado en casa me provoca ansiedad. & $\begin{array}{l}10.4 \% \\
(\mathrm{n}=10)\end{array}$ & $\begin{array}{l}27.5 \% \\
(\mathrm{n}=36)\end{array}$ & $\begin{array}{l}19.5 \% \\
(\mathrm{n}=16)\end{array}$ & $\begin{array}{l}6.5 \% \\
(\mathrm{n}=5)\end{array}$ & $49.57 * * *$ & .15 \\
\hline $\begin{array}{l}\text { No saber hasta cuando tendré que estar confinado/a en } \\
\text { casa me provoca ansiedad. }\end{array}$ & $\begin{array}{l}18.6 \% \\
(\mathrm{n}=22)\end{array}$ & $\begin{array}{l}49.3 \% \\
(\mathrm{n}=57)\end{array}$ & $\begin{array}{l}35.4 \% \\
(\mathrm{n}=31)\end{array}$ & $\begin{array}{l}11.6 \% \\
(\mathrm{n}=10)\end{array}$ & $44.80 * * *$ & .14 \\
\hline $\begin{array}{l}\text { Pienso que después de las crisis del Coronavirus mi } \\
\text { situación laboral previa a la crisis va a cambiar } \\
\text { considerablemente. }\end{array}$ & $\begin{array}{l}17.3 \% \\
(n=20)\end{array}$ & $\begin{array}{l}45.9 \% \\
(\mathrm{n}=48)\end{array}$ & $\begin{array}{l}33 \% \\
(\mathrm{n}=25)\end{array}$ & $\begin{array}{l}10.8 \% \\
(\mathrm{n}=10)\end{array}$ & $19.99 * * *$ & .10 \\
\hline
\end{tabular}

Nota $* * * p<.001 * * p<.01 ; * p<.05$

Fuente: Elaboración propia.

\subsection{Asociaciones entre las variables normas sociales, control social y medios de comunicación}

En la Tabla 6 se muestra una de las preguntas que ha señalado asociaciones estadísticamente significativas entre sí. Concretamente la asociación se ha dado entre las siguientes variables: alguna vez he increpado desde mi casa a personas que no están respetando el confinamiento y creo que los medios de comunicación informan de un modo responsable sobre el Coronavirus, $x 2(5)=14.30, p$ $=.014$, Vcramer $=.14$, con un tamaño del efecto pequeño. 
RLCS, Revista Latina de Comunicación Social, 78, 437-456

[Investigación] DOI: 10.4185/RLCS-2020-1484| ISSN 1138-5820| Año 2020

Tabla 6. Asociaciones entre variables

\begin{tabular}{lccc}
\hline & X2 (gl) & p & Vcramer \\
\hline Alguna vez he increpado desde mi casa a personas que no están respetando el & $14.30(5)$ & .014 & .14 \\
confinamiento * Creo que los medios de comunicación informan de un modo & & \\
responsable sobre el Coronavirus. & & \\
\hline *Variables asociadas entre sí
\end{tabular}

*Variables asociadas entre sí

Fuente: Elaboración propia.

\section{Discusión}

Los datos recogidos para la realización del presente estudio muestran una serie de evidencias que ponen en valor algunas de las teorías presentadas con anterioridad. Se percibe en el análisis de los datos de tipo cuantitativo que existen ciertas homogeneidades en torno a las preguntas realizadas y analizadas a partir de la variable de la edad y, en el caso de las normas sociales, también el sexo.

Teniendo en cuenta las diferencias entre hombres y mujeres, sorprende que ninguna de las preguntas directamente asociadas al control social o a los medios de comunicación hayan mostrado asociaciones estadísticamente significativas entre ambos grupos. Sin embargo, sí se detectaron tres cuestiones referidas a las normas sociales en las que la diferenciación de género era considerable, siendo las mujeres las que contestaron más afirmativamente que los hombres afirmando que el confinamiento era necesario y que el acto de recriminar comportamientos no adecuados de la población era beneficioso para combatir la pandemia. De acuerdo con Litton (1977), cabría esperar este tipo de respuestas ya que, en este contexto, las normas sociales alteran y dirigen sutilmente el comportamiento de las mujeres bajo el argumento de un beneficio y cuidado colectivo. Además, y en palabras del mismo autor, el conjunto de normas sociales que reproducen ciertos rasgos de la feminidad como puede ser la predisposición a la aceptación, la discreción o la resignación, se espera de las mujeres respondan de una forma más positiva a las normas sociales, tanto en términos de acatarlas como de ejercer control sobre otras personas a través de dichas normas. En este orden de ideas, el papel de los medios de comunicación es relevante en tanto en cuanto a la legitimación de dichas normas sociales (Juris, 2003), a través de la imagen que proyectan de la mujer y de sus responsabilidades para con la sociedad (Galarza, Cobo y Esquembre, 2020; Radl, 2011).

En cuanto a la edad, cabe destacar que los grupos centrales (26-39 años y 40-55 años) fueron los que presentaron porcentajes más elevados en lo que respecta a la percepción de las normas sociales. Además, con respecto a su percepción sobre dichas normas, afirmaron en mayor medida que el resto de grupos de edad que los políticos, policía y sociedad en general querían que denunciaran a personas que incumplían el confinamiento.

En lo que respecta a la comparación de los rangos de edad más bajos con aquellos más altos, se observan similitudes con el estudio de Useche et al., (2018), en el que se señala que las personas menores de 25 años tienen una percepción de las normas sociales más baja que aquellas más mayores. Este estudio concluye que las personas jóvenes presentan comportamientos predictores de riesgo porque perciben las normas sociales de un modo más flexible. El presente artículo sigue esta misma línea al mostrar que las personas más jóvenes perciben el control social de un modo más laxo. Dicho de otro modo, los grupos de edad centrales, es decir, aquellos entre 26-39 años y 40-55 años, percibían en un mayor grado el control que ejercen ciertas estructuras sociales como son la sociedad en general, la policía y el gobierno en la pandemia del COVID-19. De dicha percepción de control social puede derivar el hecho de que estos grupos de edad crean que controlando a la gente ayudan a la sociedad. 
En cuanto al contenido relacionado con el control social, se observan tendencias similares en las respuestas dadas por las dos franjas de edades centrales (26-39/40-55), por una parte, y por los grupos de edades de los dos extremos (18-25/>56) por otra. Esta misma tendencia se ha podido apreciar también en los resultados relativos a las normas sociales. Los datos indicarían en cierto modo que los grupos de edades centrales responderían a algunas de las lógicas relativas a los comportamientos y cosmovisiones destacadas por Laca (2011), según las cuales la prolongación y normalización de medidas de control social dictaminadas por las autoridades políticas competentes, diseminadas y legitimadas por los medios de comunicación e implementadas por los cuerpos de seguridad, derivarían en una "cultura basada en el miedo" como resultado del empleo del poder y del control social con fines determinados. Esta cultura basada en el miedo tendría similitudes manifiestas con el concepto de violencia simbólica empleado por Bourdieu (1991, 1997), quien la define como la instauración de un tipo de dominación a nivel íntimo de determinados colectivos sociales, mediante el reconocimiento por parte de estos de las estructuras de poder, colaborando además en su propia opresión cada vez que perciben y juzgan el orden social a través de categorías que hacen que éste parezca natural y evidente por sí mismo, tal y como se apuntaba anteriormente en cuanto a una mayor predisposición de las mujeres a la hora de acatar las normas sociales. Y es que, en palabras de Gitlin (1980), los gobiernos y fuerzas de seguridad podrían manipular ciertas situaciones para reinsertarlas en nuevos contextos para que así la sociedad perciba y legitime una "realidad" plagada de subjetividades. Los medios de comunicación como sistemas cardinales que son para la distribución de ideologías, comportamientos y normas (Swader, 2017), habrían tenido una mayor influencia en los rangos de edades comprendidos entre los 26-39 y los 40-55 años. El análisis de los datos también muestra que son estos dos grupos de edad los que muestran mayores índices de ansiedad y miedo al estar confinados en casa. De esta información se podría deducir que cuanto mayor sea la ansiedad y el temor ante una situación de alarma social, mayor será la normalización de medidas de control social y la asunción de estas como parte del comportamiento normalizado dentro de un grupo social o un determinado colectivo, como se recoge en estudios realizados previamente (Laca, 2011). En esta misma línea, Bowman (2001, 2003) señala que ciertas sociedades pueden actuar en sintonía con los gobiernos y las fuerzas de seguridad vigentes apoyando y legitimando las medidas que estas dictan, si se percibe que existe un peligro real o imaginario que amenaza a la propia subsistencia del grupo en cuestión.

Observamos además que los dos grupos de edad centrales (26-39 años y 40-55 años) son más críticos con el rol informativo desempeñado por los medios de comunicación durante la pandemia del COVID-19. Resulta llamativo en este sentido que si bien los dos rangos de edades centrales perciben que los medios de comunicación estarían siendo sensacionalistas en torno a la información ofrecida acerca del COVID-19 y que estos informarían en ocasiones para provocar miedo, también afirman, como veíamos anteriormente, que es necesario implementar medidas de control social más estrictas, aunque eso suponga que el Gobierno y cuerpos policiales tengan acceso a la vida personal de las personas. En este sentido, se observaría del mismo modo que afirma Juris (2003), que los medios de comunicación son efectivos instrumentos para el control y la normativización social debido a su capacidad para definir qué está bien y qué está mal en términos de normalidad sociocultural. Deberíamos traer a la palestra en este punto, una vez más, los mayores niveles tanto de ansiedad como de miedo que estos dos grupos de edad (26-39 / 40-55) experimentan ante la posibilidad de que la economía afecte a su bienestar después del confinamiento, como vienen indicando diariamente los propios medios de comunicación. Además del tratamiento sensacionalista de las noticias en general, incluyendo aquellas de carácter económico, que los dos rangos de edad centrales indican, también inciden en mayor medida que el resto de rangos de edad, en que los medios de comunicación informan tanto sobre el Coronavirus para que la sociedad sienta miedo. Estos rangos de edad vuelven a mostrar mayores índices de ansiedad y miedo ante posibles consecuencias de carácter social y en lo referente al ámbito económico. En este caso aconteceríamos a un escenario opuesto al presentado por 
Witte y Allen (2000), ya que la población en general, y sobre todo aquella que se sitúa en los rangos de edad centrales, considera que se enfrentará a graves problemas para hacer frente a las consecuencias directas de la pandemia COVID-19. Asimismo, también decrece exponencialmente su percepción sobre la competencia del Gobierno y por ende su confianza hacia este para salir airosos de la crisis. Por otro lado, los rangos de edad centrales consideran que su comportamiento tiene un impacto positivo en otras personas bien sea a través de la aceptación y legitimación de las normas sociales y leyes impuestas, como a través de su defensa o implementación. En este punto surge de nuevo la necesidad de considerar el rol informativo y normativo que desempeñan los medios de comunicación, así como su influencia sobre la población en general para imponer una cosmovisión hegemónica de la "realidad". Y es que en palabras de Juris (2008), no se debe obviar la capacidad de los medios de comunicación para normativizar mensajes y retóricas altamente performativas (Bourdieu, 1991, 1997) capaces de dictar de un modo efectivo qué comportamientos deben ser aceptados y cuáles rechazados. Lo anteriormente comentado derivaría de nuevo en la idea de que los medios de comunicación serían agentes primarios en la producción de realidad social, siempre según sus propios intereses y objetivos estratégicos.

Las asociaciones entre las variables relativas a las normas sociales, el control social y el rol de los medios de comunicación, refrendarían algunos de los argumentos planteados anteriormente, de los cuales podríamos interpretar, como sucedían en apartados previos, que existe una relación directa entre la confianza en la información dada por los medios de comunicación y la influencia de estos sobre ciertos colectivos y rangos de edad a la hora de ejercer un determinado control social sobre el resto de la población (Blair, Morse y Tsai, 2017). En este sentido, deberíamos recuperar las palabras de Juris (2003), quien incide en que los medios de comunicación tienden a reproducir significados sociales, culturales y políticos que responden a los intereses dominantes de la sociedad mediante la construcción de una serie de discursos destinados a determinar qué normas han de cumplirse y qué límites serían infranqueables.

\section{Conclusiones}

Tanto la literatura empleada como los resultados obtenidos en el presente estudio, dan fe de la profunda influencia que los medios de comunicación tendrían en los procesos de construcción de los roles sociales (Bowman, 2001, 2003; Hage 1995; Juris, 2003, 2008; Roman, 2015, 2018) y en los modos en los que las personas desarrollan sus propias cosmovisiones en torno a la "realidad" social que les rodea. La sociedad puede en ocasiones, de un modo consciente o inconsciente, acabar ejerciendo un determinado control social sobre sus conciudadanos que se derivaría de la performatividad de los mensajes articulados por los medios de comunicación. Los medios, en línea con los intereses de las fuerzas políticas y policiales, así como garantes de los intereses de los poderes económicos que acaparan la propiedad de los propios medios, pueden determinar cuáles son las normas que deben cumplirse y cuáles son los límites que nadie podrá traspasar (Gitlin, 1980).

La desinformación, la manipulación de esta, las fake news y los bulos pueden incidir sustancialmente en cómo las personas perciben e interpretan la "realidad" social y política, y consecuentemente cómo actúan. La influencia que los medios de masas tienen sobre la sociedad es notoria, y más aún en la era de la comunicación donde la información se da prácticamente a tiempo real y de un modo globalizado. Los medios están activamente comprometidos en la producción de la "realidad" social y pueden implementar retóricas y discursos dualistas orientados a que la sociedad en general acate, legitime e implemente una serie de normas sociales orientadas a la puesta en práctica y defensa de ciertos intereses particulares (Yongtao, 2010), que por lo general coinciden con aquellos intrínsecos a las clases dominantes y al poder político y policial (Herman y Chomsky, 1988). 
Este fenómeno se manifestaría en mayor medida en el seno de determinados grupos sociales, colectivos o rangos de edad a los que por distintas razones como pueden ser la ansiedad, el miedo o intereses particulares relacionados con la economía, la política, la cultura u otros componentes de carácter etnocultural (Roman, 2015), la influencia de los mensajes emitidos y distribuidos por los medios de comunicación afectarían de un modo más notorio. El sexo sería otro de los factores determinantes en la exposición y legitimación del control a través de las normas sociales asociadas a la pandemia del COVID-19 (McLaren, Wong, Nguyen y Mahamadachchi, 2020), acordadas y aprobadas por las fuerzas políticas, implementadas por las fuerzas de seguridad, y legitimadas y distribuidas masivamente por los medios de comunicación (Galarza, Cobo y Esquembre, 2016). Asimismo, y en lo relativo al rol de los medios de comunicación en la difusión de recomendaciones normativas a la vez que performativas, los datos recogidos, muestran que los rangos de edad centrales, distintamente de lo que opinan las personas más jóvenes y las más mayores, consideran necesario implementar las normas sociales orientadas a la gestión de la pandemia. Del mismo modo, inciden en la necesidad de endurecer las acciones punitivas que se impongan por su no cumplimiento. En este sentido se observó una clara conexión asociada a la edad y al sexo, entre el rol informativo desempeñado por los medios de comunicación y la influencia de estos a la hora de legitimar los postulados de las fuerzas políticas y policiales, y el nivel de ansiedad y miedo derivado del consumo de la información recibida de esos mismos medios de comunicación.

Podría interpretarse, en este sentido, que en situaciones de alarma social en las que los mecanismos habituales con que cuenta la sociedad para su correcto funcionamiento, algunos medios de comunicación implementarían estrategias discursivas y retóricas orientadas a fines concretos. Estos fines podrían estar a su vez asociados a intereses particulares que afectarían directamente a ciertos colectivos, fomentando el advenimiento y la normalización de una "cultura del miedo", que podría derivar en un futuro en el que la exclusión, la desprotección social y el miedo primen, como consecuencia del rol informativo que los medios comunicación desempeñan en sintonía con los intereses del mercado y de la clase política (Laca, 2011).

\section{Bibliografía}

Ashcroft, B., Griffiths, G. y Tiffin, H. (2006). The Postcolonial Studies Reader. Oxford: Routledge.

Austin J. L. (1982). Cómo hacer cosas con palabras: Palabras y acciones. Barcelona: Paidós.

Berasategi, N., Idoiaga, N., Dosil, M., Eiguren, A., Pikaza, M. y Ozamiz, N. (2020). Las voces de los niños y de las niñas en situación de confinamiento por el COVID-19. Bilbao: Servicio Editorial de la Universidad del País Vasco/Euskal Herriko Unibertsitatea.

Bevan, A. L. (2019). Designed for Threat: Surveillance, Mass Shootings, and Pre-emptive Design in School Architecture. Surveillance \& Society, 17(3/4), 550-564. https://doi.org/10.24908/ss.v17i3/4.7077

Blair, R. A., Morse, B. S., y Tsai, L. L. Public health and public trust: survey evidence from the Ebola virus disease epidemic in Liberia. Social Science \& Medicine, 172, 89-97. https://doi.org/0.1016/j.socscimed.2016.11.016

Bloch, M. (1975). Political Language, Oratory and Traditional Society. London: Academic Press.

Bloch, M. (1989). Ritual, history, and power: Selected papers in anthropology. London: Athlone. 
Bloch, M. (1998). How We think They think: Anthropological approaches to cognition, memory, literacy. Oxford: Westview Press.

Bourdieu, P. (1985), ¿Qué significa hablar?. Economía de los intercambios lingüísticos. Madrid: Akal.

Bourdieu, Pierre (1991). Language and Symbolic Power. Massachusetts: Harvard University Press.

Bourdieu, P. (1997). Razones prácticas sobre la teoría de la acción. Barcelona: Editorial Anagrama.

Bowman, G. (2001). The Violence in Identity. En B. Schmidt y I. Schroeder (Eds), Anthropology of Violence and Conflict (pp. 25-46). Londres, Inglaterra: Routledge.

Bowman, G. (2003). Constitutive Violence and the rethorics of identity: a comparative study of nationalist movements in the Israel-Occupied territories and former Yugoslavia. Social Anthropology, 11(3), 37-58.

Bradshaw, S. (2015). Engendering Development and Disasters. Disasters, 39, 54-75. https://doi.org/10.1111/disa.12111

Castillo, M. (2015). Miedo, control social y política criminal (tesis doctoral). Universidad de Jaén. Repositorio Institucional de la Universidad de Jaén. http://ruja.ujaen.es/jspui/handle/10953/716

Chakraborty, S. (2020). How risk perceptions, not evidence, have driven harmful policies on COVID-19. European Journal of Risk Regulation, 11, 236-239. https://doi.org/10.1017/err.2020.37

Cohen, S. (1985). Visions of social control: Crime, punishment and classification (pp. 127-143). Cambridge: Polity Press.

Cohn, S. K. (2012). Pandemics: waves of disease, waves of hate from the Plague of Athens to A.I.D.S. History Journal, 85, 535-555. https://doi.org/10.1111/j.1468-2281.2012.00603.X

Drury, J., Cocking, C. y Reicher, S. (2009). The nature of collective resilience: survivor reactions to the 2005 London bombings. International Journey of Mass Emergency Disasters, 27, 66-95.

Galarza, E., Cobo, R. y Esquembre, M. (2016). Medios y violencia simbólica contra las mujeres. Revista Latina de Comunicación Social, 71, 818-832. https://doi.org/10.4185/RLCS-2016-1122

Gau, J. M. (2015) Procedural justice, police legitimacy, and legal cynicism: a test for mediation effects, Police Practice and Research, 16(5), 402-415. https://doi.org/10.1080/15614263.2014.927766

Gausman, J. y Langer, A. (2020). Sex and Gender Disparities in the COVID-19 Pandemic. Journal of Women's Health, 29(4), 465-466. https://doi.org/10.1089/jwh.2020.8472

Giddens, A., \& Sutton, P. W. (2015). Conceptos esenciales de Sociología. Alianza editorial.

Gino, F., Norton, M. I. y Weber, R. A. (2016). Motivated Bayesians: feeling moral while acting egoistically. Journal of Economic Perspective, 30, 189-212. https://doi.org/10.1257/jep.30.3.189 
Gitlin, T. (1980). The Whole World is Watching: Mass Media in the Making and Unmaking of the New Left. Berkeley: University of California Press.

Golovina, N. (2014). La Comunicación Masiva y el Comportamiento del Consumidor. Orbis. Revista Científica Ciencias Humanas, 28, 190-198. https://www.redalyc.org/pdf/709/70930408011.pdf

Grasselli, G., Pesenti, A. y Cecconi, M. (2020). Critical Care Utilization for the COVID-19 Outbreak in Lombardy, Italy Early Experience and Forecast During an Emergency Response. American Medical Association, 323(16), 1545-1546. https://doi.org/10.1001/jama.2020.4031

Guo, Y. R., Cao, Q. D., Hong, Z. S., Tan, Y. Y., Chen, S. D., Jin, H. J., Tan, K. S., Wang, D. Y. y Yan, Y. (2020). The origin, transmission and clinical therapies on coronavirus disease 2019 (COVID-19) outbreak - an update on the status. Military Medical Research, 7(11). https://doi.org/10.1186/s40779-020-00240-0

Hage, G. (1995). The Spatial Imaginary of Nacional Practices: Dwelling-Domesticating / BeingExterminating. Sydney: Sydney University.

Igartua, J.-J.; Ortega-Mohedano, F. yArcila-Calderón, C. (2020). Communication use in the times of the coronavirus. A cross-cultural study. El profesional de la información, 29(3). 290-318. https://doi.org/10.3145/epi.2020.may.18

INE. (2019). Encuesta de Población Activa (EPA). Módulo sobre conciliación entre la vida laboral y la familiar. Notas en prensa. Recuperado de: https://www.ine.es/prensa/epa_2018_m.pdf

INE. (2020). Número de hogares monoparentales según sexo, edad y estado civil del progenitor. Instituto Nacional de Estadística. https://www.ine.es/jaxi/Datos.htm?path=/t20/p274/serie/prov/p01/\&file=01017.px\#!tabs-tabla

Juris, J. (2003). Violencia Representada e Imaginada. Jóvenes Activistas, el Black Bloc y los Medios de Comunicación en Génova. En F. Ferrandiz y C. Feixa (Eds.), Jóvenes sin Tregua: Culturas y Políticas de la Violencia (pp. 185-208). Barcelona: Anthropos Editorial.

Juris, J. (2008). Networking Futures The Movements against Corporate Globalization. London: Duke University Press.

Laca, F. A. (2011). Retorno a Hobbes: Hacia una cultura del miedo. Estudios sobre las Culturas Contemporáneas, 17(33), 9-22. https://www.redalyc.org/pdf/316/31618563002.pdf

Legido-Quigley, H., Mateos-García, J. T. Regulez, V., Gea-Sánchez, M., Muntaner, C. y McKee, M. (2020). The resilience of the Spanish health system against the COVID-19 pandemic. Lancet, 5, 251-252. https://doi.org/10.1016/S2468-2667(20)30060-8

Litton, G. (1977). "Nice Girl": Social Control of Women through a Value Construct. Journal of Women in Culture and Society, 2(4), 805-817.

McLaren, H.J., Wong, K.R., Nguyen, K.N. y Mahamadachchi, K.N.D. (2020). Covid-19 and Women's Triple Burden: Vignettes from Sri Lanka, Malaysia, Vietnam and Australia. Social Sciences, 9(87). https://doi.org/10.3390/socsci9050087. 
Naranjo, S. (2012). El derecho penitenciario: ¿La nueva cara del castigo?. En J. C. Álvarez, D. E. Valencia y S. Naranjo (Eds.), El cuerpo, el alma y la víctima (pp. 63-107). Colección Universidad de Antoquia.

Nordstrom, C. y Robben, A. (1995). Fieldwork Under Fire. Contemporary Studies of Violence and Survival. Londres: University of California Press.

Ozamiz, N., Dosil, M., Picaza., M. y Idoiaga, N. (2020). Niveles de estrés, ansiedad y depresión en la primera fase del brote del COVID-19 en una muestra recogida en el norte de España. Cadernos de Saúde Pública, 36(4). https://doi.org/10.1590/0102-311X00054020

Pakpour, A. H. y Griffiths, M. D. (2020). The fear of COVID-19 and its role in preventive behaviors. Journal of Concurrent Disorders, 2(1), 58-63. http://irep.ntu.ac.uk/id/eprint/39561

Palmer, D. y Warren, I. (2012). Tecnología de vigilancia y controles territoriales: Gobernanza y el pulso de la privacidad. Novática, 217, 15-20. http://hdl.handle.net/10536/DRO/DU:30048343

Radl, R. (2011). Medios de comunicación y violencia contra las mujeres. Elementos de violencia simbólica en el medio televisivo. Revista Latina de Sociología, 1, 156-181. https://doi.org/10.17979/relaso.2011.1.1.1199

Ramón Fernández, F. (2020). Comunicación y noticias falsas en relación al COVID-19: algunas reflexiones sobre la información, la desinformación y propuestas de mejora. Revista Espanola de Comunicacion en Salud, 253-264. https://doi.org/10.20318/recs.2020.5375

Rodríguez Andrés, R. (2018). Fundamentos del concepto de desinformación como práctica manipuladora en la comunicación política y las relaciones internacionales. Historia y Comunicación Social, 23(1), 231-244. ttps://doi.org/10.5209/HICS.59843

Rodríguez-Fernández, L. (2019a). Desinformación: retos profesionales para el sector de la comunicación. El profesional de la información, 28(3), 280-306. https://doi.org/10.3145/epi.2019.may.06

Rodríguez-Fernández, L. (2019b). Desinformación y comunicación organizacional: estudio sobre el impacto de las fake news. Revista Latina de Comunicación Social, 73. 1714-1728. http://doi.org/10.4185/RLCS-2019-1406

Roman, G. (2015). El Deseo Nacional. La Gramática del Surgimiento de los Sujetos Políticos. Lejona: Universidad del País Vasco.

Roman, G., Idoiaga, N. and Berasategi, N. (2018). Inclusion processes with refugees and migrants: best practices and strategies in leisure and free time. En Vega, M., De la Fuente, R. y Romero, G. (Ed.), Focalizando áreas del saber desde sus nuevas lecturas. Barcelona: Gedisa Editorial.

Ross, E. A. (2017). Social control: A survey of the foundations of order. Routledge.

Russell, A. (2020). The rise of coronavirus hate crimes. The New Yorker. https://www.newyorker.com/news/letter-from-the-uk/the-rise-of-coronavirus-hate-crimes 
Salaverría, R., Buslón, N., López-Pan, F., León, B., López-Goñi, I. y Erviti, M.-C. (2020). Desinformación en tiempos de pandemia: tipología de los bulos sobre la Covid-19. El profesional de la información, 29(3). 290-315. https://doi.org/10.3145/epi.2020.may.15

Sheikh, A., Sheikh, Z. y Sheikh, A. (2020). Novel approaches to estimate compliance with lockdown measures in the COVID-19 pandemic. Viewpoints, 10(1), 1-4. https://doi.org/10.7189/jogh.10.010348

Sibley, C. G., Greaves, L. M., Satherley, N., Wilson, M. S., Overall, N. C., Lee, C. H. J., Milojev, P., Bulbulia, J., Osborne, D., Milfont, T. L., Houkamau, C. A., Duck., I. M., Vickers-Jones, R. y Barlow, F. K. (2020). Effects of the COVID-19 pandemic and nationwide lockdown on trust, attitudes towards government, and wellbeing. American Psychologist. https://doi.org/10.1037/amp0000662

Swader, C. (2017). Modernization, formal social control, and anomie: A 45-society multilevel analysis. International Journal of Comparative Sociology, 58(6), 494-514. https://doi.org/10.1177/0020715217736556

Useche, S., Alonso, F., Montoro, L. y Esteban, C. (2018). Explaining self-reported traffic crashes of cyclists: An empirical study based on age and road risky behaviors. Safety Science, 113, 105-114. https://doi.org/10.1016/j.ssci.2018.11.021

Van Bavel, J. J., Baicker, K., Boggio, P. S., Capraro, V., Cichocka, A., Cikara, M.,... Willer, R. (2020). Using social and behavioural science to support COVID-19 pandemic response. PERSPECTIVE: Nature Human Behaviour, 4, 460-471. https://doi.org/10.1038/s41562-020$\underline{0884-\mathrm{Z}}$

Wang, C., Horby, P. W., Hayden, F. G. y Gao, G. F. (2020). A novel coronavirus outbreak of global health concern. Lancet, 395, 470-473. https://doi.org/10.1016/S0140-6736(20)30185-9

Wenham, C., Smith, J. y Morgan, R. (2020). COVID-19: the gendered impacts of the outbreak. The Lancet, 395, 846-847. https://doi.org/10.1016/

Witte, K. y Allen, M. (2000). A meta-analysis of fear appeals: implications for effective public health campaigns. Health Education \& Behaviour, 27(5), 591-615. https://doi.org/10.1177/109019810002700506

Yongtao, L. (2010). Discourse, Meanings and IR Studies: Taking the Rethoric of "Axis of Evil" as a Case. Guadalajara: Fudan University.

Yuan, J., Li, M., Lv, G. y Lu, Z. K. (2020). Monitoring transmissibility and mortality of COVID-19 in Europe. International Journal of Infectious Diseases, 95, 311-315. https://doi.org/10.1016/j.ijid.2020.03.050

\section{AUTOR/AS:}

\section{Gorka Roman Etxebarrieta}

Doctor en Ciencia Política por la Universidad del País Vasco (UPV/EHU) y profesor en el Departamento de Didáctica de la Lengua y la Literatura en la Facultad de Educación de esta misma universidad. Doctorando en Psicodidáctica. Tiene diversas licenciaturas en Filología Vasca, Filología 
Inglesa y Antropología Social y Cultural. Ha realizado diversas estancias de investigación en Kent University (Reino Unido), en la Universidad de Bayona, en el Instituto Universitario de Lisboa y en Columbia University (New York). Entre sus ámbitos de interés destacan el análisis de discurso, la construcción de la identidad política y lingüística, y los procesos de inclusión socioeducativa. Forma parte de diversos proyectos de investigación internacionales y es investigador principal de un proyecto Erasmust. Es editor principal de diversos libros sobre innovación educativa e inclusión socioeducativa.

gorka.roman@ehu.eus

Índice H: 2

Orcid ID: https://orcid.org/0000-0002-5826-2797

Google Scholar: https://scholar.google.com/citations?user=t7C7FNIAAAAJ\&hl=es

ResearchGate: https://www.researchgate.net/profile/Gorka_Roman

Academia.edu: Gorka Roman | University of the Basque Country, Euskal Herriko Unibertsitatea

\section{María Álvarez-Rementería Álvarez}

Doctoranda del programa de Psicodidáctica de la Universidad del País Vasco (UPV/EHU). Ha estudiado un Máster en Psicodidáctica (UPV/EHU) y tiene un grado en Magisterio de Educación Primaria (UCM). Desde comienzos de 2020 es miembro de KideON, grupo de investigación consolidado del Gobierno Vasco. Sus principales líneas de investigación giran en torno a la Educación Inclusiva, la formación continua del profesorado y la inclusión sociocultural. En 2019 disfrutó de una estancia en la National University of Ireland (NUI - Galway).

maria.alvarezrementería@ehu.eus

Orcid ID: https://orcid.org/0000-0001-8033-6150

ResearchGate: https://www.researchgate.net/profile/Maria_Alvarez-Rementeria_Alvarez

\section{Elizabeth Pérez-Izaguirre}

Elizabeth Pérez Izaguirre es diplomada en Educación Social y licenciada en Antropología Social y Cultural (UPV/EHU). En 2013 finalizó un Máster en Relaciones Internacionales (University of Kent) y en 2018 defendió su tesis Education, language, and identity in multi-ethnic environments: a case study (UPV/EHU). Actualmente, es profesora adjunta del departamento de Didáctica y Organización Escolar. Desarrolla su labor utilizando metodología cualitativa y cuantitativa y ha realizado investigación etnográfica en la escuela vasca para estudiar la identidad del alumnado inmigrante y la diversidad lingüística, la relación entre docentes y alumnado, tomando en consideración las relaciones de poder. También ha realizado estancias de investigación en la Case Western Reserve University (CWRU) y la Universidad de Nevada, Reno (UNR).

elizabeth.perez@ehu.eus

\section{Índice H: 2}

Orcid ID: https://orcid.org/0000-0003-4787-6723

Google Scholar: https://scholar.google.es/citations?user=skaLS_sAAAAJ\&hl=es

ResearchGate: https://www.researchgate.net/profile/Elizabeth_Perez_Izaguirre

Academia.edu: https://easynet.academia.edu/ElizabethP\%C3\%A9rezIzaguirre

\section{Maria Dosil Santamaria}

Doctora en Psicología. Graduada en Educación Social y especialista en mediación. Consta de experiencia en el sector social y de varias publicaciones sobre personas adultas. Forma parte de proyectos de innovación y de proyectos internacionales.

maria.dosil@ehu.eus

Orcid ID: https://orcid.org/0000-0002-8805-9562

ResearchGate: https://www.researchgate.net/profile/Maria_Santamaria10 